\title{
Tumor mutation burden as emerging biomarker in non-small cell lung cancer and beyond
}

\author{
Rieke N. Fischer, Jürgen Wolf \\ Lung Cancer Group Cologne, Department I for Internal Medicine, University Hospital of Cologne, Cologne, Germany \\ Correspondence to: Jürgen Wolf. Lung Cancer Group Cologne, Department I for Internal Medicine, University Hospital of Cologne, Cologne, \\ Germany. Email: juergen.wolf@uk-koeln.de. \\ Comment on: Rizvi H, Sanchez-Vega F, La K, et al. Molecular Determinants of Response to Anti-Programmed Cell Death (PD)-1 and Anti- \\ Programmed Death-Ligand 1 (PD-L1) Blockade in Patients With Non-Small-Cell Lung Cancer Profiled With Targeted Next-Generation \\ Sequencing. J Clin Oncol 2018;36:633-41.
}

Submitted Jul 20, 2018. Accepted for publication Aug 02, 2018.

doi: $10.21037 /$ tcr.2018.08.09

View this article at: http://dx.doi.org/10.21037/tcr.2018.08.09

Tumor mutation burden (TMB) has shown to be predictive for response to immune checkpoint inhibition in several tumor entities, including non-small cell lung cancer (NSCLC) $(1,2)$. However, implementation of TMB for treatment decision is hampered by the fact that measurement of whole exome sequencing (WES) is not feasible for clinical routine.

In their work, Rizvi et al. provide the valuable evidence that TMB measured by targeted next-generation sequencing (NGS) correlates with TMB assessed by WES. Furthermore, they were able to show an increase in durable clinical benefit (DCB) and in progression-free survival (PFS) for NSCLC patients with levels above the $50^{\text {th }}$ percentile of TMB. In line with other reports (3), programmed deathligand 1 (PD-L1) expression and TMB levels did not correlate, but the combination of both values resulted in a higher probability of treatment benefit compared to each alone. This study gives another hint for the unmet need to determine biomarkers beyond PD-L1 in order to more accurately preselect patients with benefit from immune checkpoint inhibition.

Current treatment strategies yield to optimize response rates while balancing the risk of therapy-related toxicity. The programmed death-1 (PD-1) antibody pembrolizumab has proven efficacy in patients with high PD-L1 expression on their tumor cells (TC) and thus is the current standard therapy for first-line patients with PD-L1 expression of $\geq 50 \%$ TC (4). In patients with PD-L1 expression below $50 \%$, pembrolizumab failed to prove a clear advantage over chemotherapy concerning overall response rate (ORR), PFS and overall survival (OS) (5).

As the KEYNOTE-021 phase 2 trial recruiting independent from PD-L1 status showed superiority for pembrolizumab in combination with pemetrexed/ carboplatin in terms of response rates and PFS compared to chemotherapy alone, the US Food and Drug Administration (FDA) granted accelerated approval to the pembrolizumab/ chemotherapy combination (6). These data were confirmed by the phase 3 KEYNOTE-189 trial which additionally showed clear advantages in OS in the total study population, but also in subgroups defined by PD-L1 status (PD-L1 <1\%, 1-49\%, $\geq 50 \%$ ) (7). Based on KEYNOTE-189, chemotherapy-pembrolizumab combination is now regarded as standard first line treatment in patients with PD-L1 $<50 \%$ and approval can soon be expected also by the European Medicines Agency (EMA).

However, these data focus only on PD-L1 expression for patient selection, which has some limitations in terms of accuracy. Non-responses of around 30\% in pembrolizumab/ chemotherapy patients and around 50\% in PD-L1 high patients treated with pembrolizumab monotherapy underline the need to implement more specific biomarkers.

TMB has first emerged as biomarker in the CheckMate 032 trial. This is a phase I/II study of first line advanced disease small cell lung cancer (SCLC) patients treated either with nivolumab alone or in combination with ipilimumab. TMB data were available in 211 patients. PFS and OS were significantly longer in TMB high patients, but this effect could only be observed in patients who were treated with the combination therapy. Median OS reached 22 months 
in TMB high patients receiving nivolumab and ipilimumab with a 1 -year OS rate of $62.4 \%$ (8). Given the poor prognosis of SCLC with median OS of less than 10 months for patients with extensive disease in historical data (9) these results are remarkable. Nivolumab alone resulted in a median OS of 5.4 months and a 1 -year OS rate of $35.2 \%$ in TMB high patients. This difference in the clinical outcome between nivolumab monotherapy and nivolumab/ ipilimumab combination therapy might indicate, that higher mutational load correlates with a higher probability of expression of immunogenic antigens. Cytotoxic T lymphocyte-associated antigen-4 (CTLA-4) blockade by ipilimumab thus might promote remodeling of the $\mathrm{T}$ cell repertoire leading to an increase in repertoire diversity with subsequent higher probability to develop $\mathrm{T}$ cell clones reactive to tumor neo-antigens (9).

In first line NSCLC patients, TMB was first shown to be a predictive biomarker in the CheckMate 026 trial, which failed to demonstrate a benefit in clinical outcome for nivolumab compared to first-line chemotherapy, even in the PD-L1 high subgroup. However, a retrospective analysis of TMB revealed a significant increase in ORR and PFS in TMB high patients in the nivolumab arm compared to the chemotherapy arm. An advantage in OS could not be shown, likely due to the high cross-over rate.

Recently, data from the CheckMate 227 trial were presented which evaluated nivolumab in combination with ipilimumab $(\mathrm{IO}+\mathrm{IO})$ or in combination with chemotherapy (IO + chemotherapy) compared to chemotherapy alone. In TMB high patients, the IO + IO combination enhanced ORR and significantly prolonged PFS. This effect was independent of PD-L1 expression. In TMB high patients with $<1 \%$ PD-L1 expression, IO + IO prolonged PFS and duration of response (DOR) compared to IO + chemotherapy or chemotherapy alone (10). In TMB low patients with $<1 \%$ PD-L1 expression, PFS was similar between the three different treatment arms. Thus, TMB testing might be clinically relevant for selecting patients for IO + IO combination therapy in PD-L1 low expressers (11).

Currently the most beneficial treatment approach for firstline patients with $<50 \%$ PD-L1 expression remains an open question. Patients with low TMB and PD-L1 expression $<1 \%$ do not benefit from IO + IO or IO + chemotherapy compared to chemotherapy alone in the nivolumab/ipilimumab trial. In contrast, pembrolizumab + chemotherapy provided benefit for PD-L1 low patients, but using a different PD-L1 cutoff $(<50 \%)$ and in turn, no TMB evaluation was performed.
To date, different trial designs, cut-off values, biomarker evaluations and not least the different treatment regimens do not allow to unequivocally define the best treatment option. It might be a rational approach to consider patient individual aspects. The crossing of the PFS curves in TMB high patients within the CheckMate 227 trial shows that a substantial amount of patients experience rapid disease progression. This treatment approach thus might not be optimal for patients with a high need for quick response. The relatively long median time to treatment response of 2.7 months with IO + IO might lead to early loss of such patients. On the other side, as generally observed in immune checkpoint inhibition trials, the plateau development underlines the long lasting DOR in patients who derive treatment benefit. Additionally, the side effect profile is generally in favor for $\mathrm{IO}+\mathrm{IO}$ combination compared to chemotherapy. Furthermore, after disease progression on IO + IO first line therapy, second line chemotherapy provides a rational approach, while an effective second line treatment strategy after failure of the pembrolizumab/chemotherapy combination so far has not been defined. Given that to date the $\mathrm{IO}+\mathrm{IO}$ combination is not yet approved, patients with $<50 \% \mathrm{PD}-\mathrm{L} 1$ expression should be treated with the pembrolizumab/chemotherapy combination. As approval can be expected in future, randomized, TMB-stratified trials are warranted to allow a rational selection of the most effective therapeutic regimens.

In contrast to the PD-L $1<50 \%$ group, first-line pembrolizumab monotherapy is well established in the PD-L $1 \geq 50 \%$ patient population. However, given the ORR of $44.8 \%$ for first-line pembrolizumab, non-response of more than half of this patient population warrants further improvement. As PD-L1 expression and TMB don't seem to correlate, it is likely that in this patient population some patients might benefit from addition of ipilimumab in order to enhance response rates. In conclusion, TMB testing should not focus on tumors with PD-L1 $<50 \%$ expression only, but rather include all firstline patients independent of PD-L1 status.

It is evident, that clinical trials which prospectively evaluate TMB are urgently needed, not only in NSCLC but in all tumor entities. While PD-L1 expression as predictive biomarker is very heterogeneous and thus only plays a role for clinical treatment decision in a minority of tumor entities, TMB possibly is a biomarker which might be transferred to a wide range of tumors. As cases of high TMB can be identified in almost every tumor entity (12) it might in future even lead to a new tumor classification based 
on mutation burden. This is underlined by the impressive effect of immune checkpoint inhibition in microsatellite instability (MSI)-high or mismatch repair (MMR)-deficient solid tumors which lead to approval of pembrolizumab in this patient population by the FDA. These tumors are characterized by high levels of somatic mutations, regardless of the cells of origin (13). The subsequently generated large numbers of mutation-associated neoantigens (MANAs) are highly immunogenic. It can be concluded, that TMB high and MSI-high/MMR-deficient tumors reveal the same mechanism of activation of tumor-antigen specific $\mathrm{T}$ cells with higher amount of immune cell infiltration in the tumor microenvironment.

In order to use TMB in clinical routine, affordable, standardized methods which provide testing results in a reasonable period of time need to be implemented. This first evidence from Rizvi et al. for NGS-based TMB analysis is therefore very promising for future approaches for prospective evaluation. Similar to PD-L1 expression, different cut-off levels have been chosen and to date none has been tested in a prospective manner. Thus, the $50^{\text {th }}$ percentile cut-off selected in this work warrants future evaluation. Also, it is not obvious, why DCB was defined by the 6 months threshold. Five-year OS updates from early immune checkpoint inhibitor trials (Gettinger, $7 C O$ 2018) are promising and future biomarker approaches should focus on the identification of such patients with a meaningful long-term benefit.

Nevertheless, the retrospective analysis performed by Rizvi et al. on patients treated in their institute provides first evidence that this biomarker is applicable on a realworld collective which is not pre-selected for inclusion into a clinical trial. This work underlines the relevance of collecting biomaterial together with clinical data from patients treated outside of clinical trials for exploratory analysis. That approach could help to assess combinations of different biomarkers which will eventually allow making rational treatment decisions in this increasingly complex and rapidly developing therapeutic landscape.

In conclusion, Rizvi et al. provide robust evidence, that TMB is a predictive biomarker for treatment response to immune checkpoint inhibition which can be assessed by NGS in clinical routine. It is very likely that TMB will soon be established as a routine biomarker with strong potential to influence therapeutic strategies and thus will lead to a substantial change in treatment decisions, not only in NSCLC patients, but possibly in a large group of malignant tumors.

\section{Acknowledgments}

Funding: None.

\section{Footnote}

Provenance and Peer Review: This article was commissioned and reviewed by the Section Editor Dr. Wei Xu (Jiangsu Provincial Key Laboratory of Geriatrics, Department of Geriatrics, the First Affiliated Hospital with Nanjing Medical University, Nanjing, China).

Conflicts of Interest: J Wolf: the advisory boards and lecture fees was supported by Abbvie, AstraZeneca, BMS, Boehringer-Ingelheim, Chugai, Ignyta, Lilly MSD, Novartis, Pfizer, Roche. Research was supported by BMS and MSD, Novartis, Pfizer. RN Fischer: the advisory boards and lecture fees was supported by BMS, MSD, Roche, Boehringer-Ingelheim and the research was supported by BMS and MSD.

Etbical Statement: The authors are accountable for all aspects of the work in ensuring that questions related to the accuracy or integrity of any part of the work are appropriately investigated and resolved.

Open Access Statement: This is an Open Access article distributed in accordance with the Creative Commons Attribution-NonCommercial-NoDerivs 4.0 International License (CC BY-NC-ND 4.0), which permits the noncommercial replication and distribution of the article with the strict proviso that no changes or edits are made and the original work is properly cited (including links to both the formal publication through the relevant DOI and the license). See: https://creativecommons.org/licenses/by-nc-nd/4.0/.

\section{References}

1. Rizvi NA, Hellmann MD, Snyder A, et al. Cancer immunology. Mutational landscape determines sensitivity to PD-1 blockade in non-small cell lung cancer. Science 2015;348:124-8.

2. Carbone DP, Reck M, Paz-Ares L, et al. First-Line Nivolumab in Stage IV or Recurrent Non-Small-Cell Lung Cancer. N Engl J Med 2017;376:2415-26.

3. Peters S, Creelan B, Hellmann MD, et al. Abstract CT082: Impact of tumor mutation burden on the efficacy of firstline nivolumab in stage IV or recurrent non-small cell lung 
cancer: An exploratory analysis of CheckMate 026. Cancer Res 2017;77:abstr nr CT082.

4. Reck M, Rodríguez-Abreu D, Robinson AG, et al. Pembrolizumab versus Chemotherapy for PD-L1Positive Non-Small-Cell Lung Cancer. N Engl J Med 2016;375:1823-33.

5. Lopes G, Wu YL, Kudaba I, et al. Pembrolizumab vs platinum-based chemotherapy as first-line therapy for advanced/metastatic NSCLC with a PD-L1 TPS $\geq 1 \%$ : open-label, phase 3 KEYNOTE-042 study. J Clin Oncol 2018. Available online: http://abstracts.asco.org/214/ AbstView_214_226263.html

6. Langer CJ, Gadgeel SM, Borghaei H, et al. Carboplatin and pemetrexed with or without pembrolizumab for advanced, non-squamous non-small-cell lung cancer: a randomised, phase 2 cohort of the open-label KEYNOTE-021 study. Lancet Oncol 2016;17:1497-508.

7. Gandhi L, Rodríguez-Abreu D, Gadgeel S, et al. Pembrolizumab plus Chemotherapy in Metastatic NonSmall-Cell Lung Cancer. N Engl J Med 2018;378:2078-92.

8. Rizvi N. Impact of Tumor Mutation Burden on the Efficacy of Nivolumab or Nivolumab + Ipilimumab in Small Cell Lung Cancer: An Exploratory Analysis of CheckMate 032. Available online: https://

Cite this article as: Fischer RN, Wolf $\mathrm{J}$. Tumor mutation burden as emerging biomarker in non-small cell lung cancer and beyond. Transl Cancer Res 2018;7(Suppl 7):S770-S773. doi: 10.21037/tcr.2018.08.09 library.iaslc.org/conference-program?product $\mathrm{id}=7 \&$ author $=\&$ category $=\&$ date $=2017-10-16 \&$ session_ty pe $=\&$ session $=\&$ presentation $=\&$ keyword $=$ Rizvi $\&$

9. Foster NR, Qi Y, Shi Q, et al. Tumor response and progression-free survival as potential surrogate endpoints for overall survival in extensive stage small-cell lung cancer: findings on the basis of North Central Cancer Treatment Group trials. Cancer 2011;117:1262-71.

10. Hellmann MD, Ciuleanu TE, Pluzanski A, et al. Nivolumab plus Ipilimumab in Lung Cancer with a High Tumor Mutational Burden. N Engl J Med 2018;378:2093-104.

11. Borghaei H, Hellmann MD, Paz-Ares LG, et al. Nivolumab + Ipilimumab, Nivolumab + Chemotherapy, and Chemotherapy in Chemo-Naive Patients With Advanced Non-Small Cell Lung Cancer and $<1 \%$ Tumor PD-L1 Expression: Results From CheckMate 227. Available online: http://abstracts.asco.org/214/ AbstView_214_229709.html

12. Chalmers ZR, Connelly CF, Fabrizio D, et al. Analysis of 100,000 human cancer genomes reveals the landscape of tumor mutational burden. Genome Med 2017;9:34.

13. Le DT, Durham JN, Smith KN, et al. Mismatch repair deficiency predicts response of solid tumors to PD-1 blockade. Science 2017;357:409-13. 